\title{
Structure-guided discovery of dual recognition chemibodies
}

\author{
Alan C. Cheng, Elizabeth M. Doherty, Sheree Johnstone, Erin F. DiMauro, Jennifer \\ Dao, Abhinav Luthra, Jing Ye, Jie Tang, Thomas Nixey, Xiaoshan Min, Philip \\ Tagari, Les P. Miranda*, Zhulun Wang*
}

Amgen Inc. 1120 Veterans Blvd. South San Francisco, CA 94080

Correspondence: lesm@amgen.com or zwang@amgen.com

Small molecules and antibodies each have advantages and limitations as therapeutics. Here, we present for the first time to our knowledge, the structure-guided design of "chemibodies" as small molecule-antibody hybrids that offer dual recognition of a single target by both a small molecule and an antibody, using DPP-IV enzyme as a proof of concept study. Biochemical characterization demonstrates that the chemibodies present superior DPP-IV inhibition compared to either small molecule or antibody component alone. We validated our design by successfully solving a co-crystal structure of a chemibody in complex with DPP-IV, confirming specific binding of the small molecule portion at the interior catalytic site and the Fab portion at the protein surface. The discovery of chemibodies presents considerable potential for novel therapeutics that harness the power of both small molecule and antibody modalities to achieve superior specificity, potency, and pharmacokinetic properties.

Reference:

Cheng AC, Doherty EM, Johnstone S, DiMauro EF, Dao J, Luthra A, Ye J, Tang J, Nixey T, Min X, Tagari P, Miranda LP, Wang Z. Structure-guided Discovery of Dual-recognition Chemibodies. Sci. Reports. 2018 15;8(1):7570. 